\title{
ANÁLISE COMPARATIVA DOS CASOS DE ALEITAMENTO MATERNO EXCLUSIVO ENTRE BRASIL E SÃO PAULO NO PERÍODO DE 2010-2015
}

\author{
Ilanna Sobral de Luna, Daniele Pereira de Souza Kronka, Nicole Álik Kitamura \\ Universidade do Oeste Paulista - UNOESTE, Faculdade de Medicina, Presidente Prudente, SP. E-mail: \\ ilaanna_sobral@hotmail.com
}

\section{RESUMO}

O aleitamento materno é um processo que promove uma interação intensa entre mãe e filho e repercute no estado nutricional do bebê. $O$ objetivo do presente estudo foi realizar análise descritiva sobre os casos de aleitamento materno exclusivo entre Brasil e o estado de São Paulo no período de 2010-2015. Trata-se de um estudo ecológico, cujos dados foram obtidos por meio da pesquisa no Sistema de Informação de Atenção Básica (SIAB). O Brasil apresentou uma redução maior nos casos de aleitamento materno exclusivo em crianças menores de 4 meses do que no estado de São Paulo. Uma possível justificativa para este padrão observado pode se dar devido a razões sociais, econômicas e culturais, sendo necessárias novas abordagens que valorizem ações mais efetivas na promoção, proteção das gestantes e apoio ao aleitamento materno.

Palavras-chave: aleitamento materno, maternidade, lactente, leite humano, período pós-parto.

\section{COMPARATIVE ANALYSIS OF EXCLUSIVE BREASTFEEDING CASES IN BRAZIL AND SÃO PAULO IN THE PERIOD 2010-2015}

\begin{abstract}
Breastfeeding is a process that promotes an intense interaction between mother and child and affects the nutritional status of the baby. The objective of the present study was to perform a descriptive analysis on the cases of exclusive breastfeeding between Brazil and the state of São Paulo in the period 2010-2015. It is an ecological study, whose data were obtained through the research in the Basic Attention Information System (SIAB). Brazil presented a greater reduction in cases of exclusive breastfeeding in children younger than 4 months than in the state of São Paulo. A possible justification for this observed pattern can be due to social, economic and cultural reasons, 7 and new approaches are needed that value more effective actions in the promotion, protection of pregnant women and support to breastfeeding.
\end{abstract}

Keywords: breast feeding, maternity, infant, milk, human, postpartum period.

\section{INTRODUÇÃO}

O aleitamento materno é um processo que promove uma interação intensa entre mãe e filho e repercute no estado nutricional do bebê, em sua fisiologia, em seus mecanismos de defesa contra as infecções, em seu desenvolvimento cognitivo e emocional, além de influenciar na saúde psíquica e física da mãe. Por essa importância a Organização Mundial de Saúde recomenda que o aleitamento materno exclusivo deve prosseguir até o sexto mês e precisa ser complementado até os 2 anos de idade ${ }^{1}$.
Além da composição balanceada de nutrientes, o leite materno possui outros componentes atuantes na defesa do organismo do lactente, como anti-inflamatórios, imunoglobulinas e imunoestimuladores. Seus mecanismos abrangem atividade específica contra agentes infecciosos, crescimento celular da mucosa intestinal aumentando a resistência às infecções, dentre outros. Existem relatos de aproximadamente 250 elementos de proteção no leite humano e também de fatores de crescimento do trato gastrintestinal' ${ }^{1}$. 
No Brasil, são desenvolvidas políticas de proteção, promoção e apoio ao aleitamento materno como: Iniciativa Hospital Amigo da Criança, Rede Brasileira de Bancos de Leite Humano, Proteção Legal ao Aleitamento Materno, Mobilização Social, Monitoramento dos Indicadores de Aleitamento Materno e a Estratégia Amamenta e Alimenta Brasil. Apesar da importância e dos benefícios do aleitamento materno, somente $37 \%$ das crianças são amamentadas exclusivamente no mundo. Devido aos avanços dessas políticas no Brasil, a duração dessa prática tem obtido melhoras, como mostram os seguintes índices: de $3,1 \%$ do aleitamento materno exclusivo em 1980 para $38,6 \%$ em 2006; e de 9,9 meses para 11,3 no período de 1999 a 2008, com incremento de pouco mais de um mês na duração mediana do aleitamento materno ${ }^{2}$.

0 presente estudo justifica-se, pois o desmame precoce ainda é um problema causado pela desinformação da população em geral e, principalmente, a dos profissionais da área de saúde. Segundo Rego ${ }^{3}$, o percentual de difusão de informações errôneas se assemelha ao percentual de mães que abandonam a amamentação alegando que "o leite não sustenta". Isso evidencia ainda mais a necessidade da capacitação de profissionais de saúde para aumentar a prevalência do aleitamento materno ${ }^{4}$.

Assim, o objetivo do presente estudo foi realizar análise descritiva dos casos de aleitamento materno exclusivo no Brasil e no estado de São Paulo no período de 2010-2015, contribuindo para políticas públicas de saúde materno-infantil a nível nacional e estadual e subsidiando informações para o planejamento em saúde.

\section{METODOLOGIA}

Trata-se de um estudo ecológico, cujos dados foram obtidos na base de dados Sistema de Informação de Atenção Básica (SIAB), disponibilizados pelo Departamento de Informática do Sistema Único de Saúde (DATASUS). Os dados coletados sobre o aleitamento materno exclusivo em crianças menores de 4 meses no período de 2010 a 2015, no Brasil e no estado de São Paulo, serviram como base para análise. Estes estão apresentados em figuras mostrando as frequências absolutas. As análises estatísticas foram realizadas por meio do teste do quiquadrado, teste exato de Fisher ou teste $G$ utilizando o programa BioEstat 5.3 e nível de significância de $p<0,05$.

\section{RESULTADOS}

Diante dos dados coletados que apresentam os respectivos valores referentes ao aleitamento materno exclusivo em crianças de até 4 meses, verificou-se que nacionalmente houve uma redução proporcional, mais acentuada do que no estado de São Paulo. A Figura 1 evidencia que no período de 2010-2015 houve decréscimo de $45,31 \%$ dos casos de aleitamento materno exclusivo no Brasil. Também se observa que no período analisado houve decréscimo de $25,26 \%$ dos casos de aleitamento materno exclusivo em São Paulo.

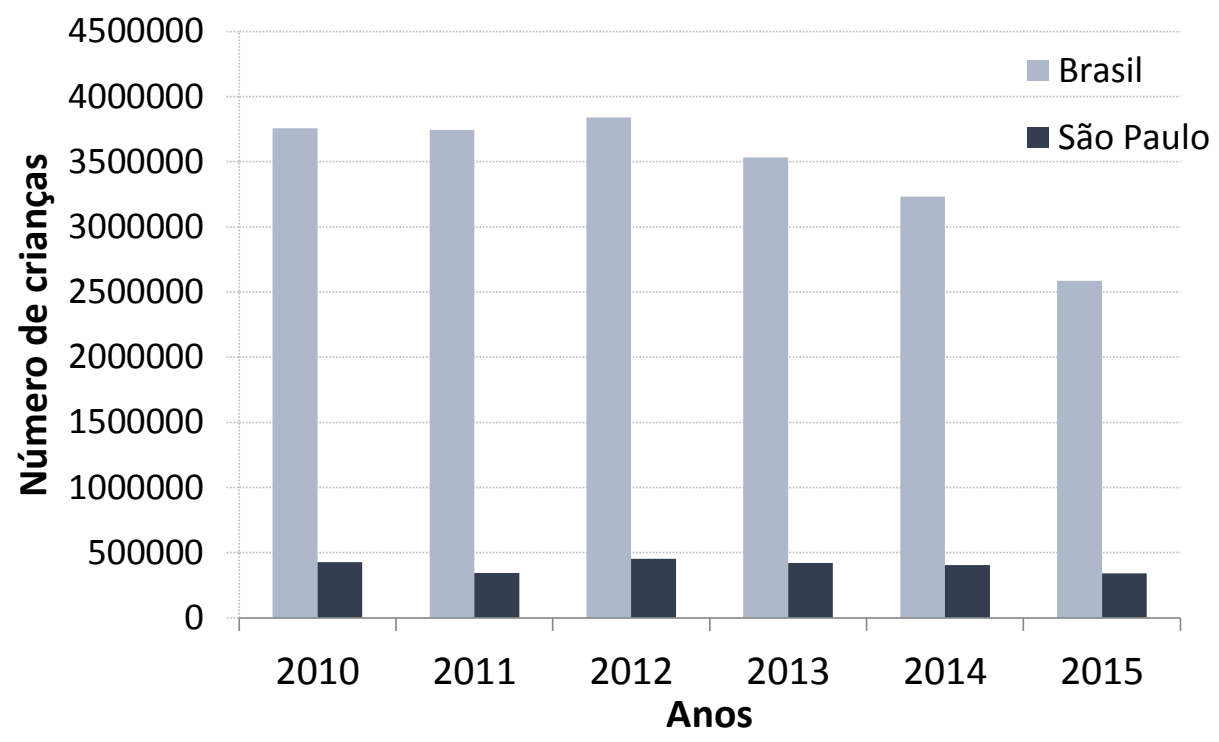

Figura 1. Aleitamento materno exclusivo no Brasil e em São Paulo no período de 2010-2015. 


\section{DISCUSSÃO}

Neste estudo, segundo os dados coletados e os resultados obtidos nota-se que a nível nacional, o Brasil apresentou uma redução no número do aleitamento materno exclusivo em crianças menores de 4 meses de $45,31 \%$ entre 2010 e 2015 e no estado de São Paulo a realidade assemelha-se uma vez que no período em análise houve uma redução de $25,26 \%$ dos casos, sendo que esses resultados se encontram aquém das recomendações internacionais ${ }^{5}$.

Com a introdução de novas tecnologias, a inserção da mulher brasileira cada vez mais frequente no mercado de trabalho e a adoção de novos estilos de vida da população do país e do estado paulista houve uma redução notável na importância atribuída a essa prática tradicional ${ }^{6}$. Uma possível justificativa para este padrão observado pode se dar devido a razões sociais, econômicas e culturais ${ }^{7}$.

A taxa de analfabetismo entre brasileiros com 15 anos ou mais em 2014 foi estimada em 13,2 milhões de pessoas, segundo a Pesquisa Nacional por Amostra de Domicílios (Pnad), realizada pelo Instituto Brasileiro de Geografia e Estatística (IBGE) ${ }^{8}$. Um estudo de coorte realizado por Bueno ${ }^{8}$ em São Paulo constatou que a escolaridade materna até o ensino fundamental representa o dobro do risco em relação ao nível superior para introdução de outros alimentos antes dos seis meses. Evidenciando assim, que ao obter conhecimento sobre os benefícios do aleitamento materno exclusivo a mulher de escolaridade mais alta venha a fazer a opção pelo aleitamento materno exclusivo nos primeiros seis meses de vida do bebê.

Também analisando o panorama social do país e do estado em estudo, observou-se que o número de divórcios no país cresceu mais de $160 \%$ na última década. Dados da pesquisa Estatísticas do Registro Civil 2014, divulgados hoje pelo IBGE, indicam que, no ano de 2014, foram homologados 341,1 mil divórcios, um salto significativo em relação a 2004, quando foram registrados 130,5 mil divórcios. Segundo um estudo realizado em países da América Latina sinalizou que, no Brasil, a duração do aleitamento materno exclusivo tendia a ser maior quando o pai do bebê morava com a família $(p=0,08)^{9}$.

Além disso, algumas crenças e mitos propagados por muitas mães que amamentam podem contribuir para os achados nesse estudo.
Uma pesquisa realizada na cidade brasileira de Perdizes constatou que $74,7 \%$ das mulheres acreditam que possuem pouco leite e $36 \%$ afirmam que seu leite é fraco e por isso não veem vantagens na amamentação exclusiva. Outro fator crucial para a atual redução dos níveis de amamentação exclusiva é o fator estético. Esse mesmo estudo obteve resultados de que 16,9\% das mulheres acham que amamentar faz a mama ficar flácida ${ }^{10}$.

Devido à redução da amamentação exclusiva nacional e no estado de São Paulo, muitas iniciativas vêm sendo desenvolvidas a fim de priorizar as práticas de saúde, com enfoque na ótica do cuidado em saúde, valorizando a escuta qualificada, o vínculo e a responsabilização na organização da assistência na atenção básica as gestantes, puérperas e mães de lactantes até os 6 meses de vida. Quase todas as mulheres são capazes de amamentar, mas para isso se faz necessário atuação multidisciplinar de profissionais de saúde realizando atividades que estimulem e orientem essas mulheres e erradiquem os mitos e mostrem que os benefícios da amamentação são fundamentais para seu filho ${ }^{11}$.

\section{CONCLUSÃO}

Conclui-se assim, que o decréscimo encontrado na média de aleitamento materno exclusivo no Brasil e no estado de São Paulo aponta que novas abordagens devem ser pensadas, valorizando ações mais efetivas na promoção, proteção das gestantes e apoio ao aleitamento materno. Esses resultados também sinalizam a qualidade da produção do cuidado à mãe com crianças lactantes assistidas na rede básica de saúde.

\section{CONFLITO DE INTERESSES}

Os autores declaram não haver qualquer potencial conflito de interesse que possa interferir na imparcialidade deste trabalho científico.

\section{REFERÊNCIAS}

1. Passanha A, Cervato-Mancuso AM, Silva MEMP. Elementos protetores do leite materno na prevenção de doenças gastrintestinais e respiratórias. Rev Bras Cresc Desenv Hum. 
2010;20(2):351-60.

https://doi.org/10.7322/ihgd.19972

2. Moreira LA, Velasco e Cruz N, Linhares FMP, Guedes TG, Martins FDP, Pontes CM. Support to woman/nourisher in the advertising pieces of the World Breastfeeding Week. Rev Bras Enferm. 2017;70(1):55-64.

DOI: http://dx.doi.org/10.1590/0034-7167-2016-0376

3. Rego JD. Amamentando um prematuro. In: Rego JD. Aleitamento materno: um guia para pais e familiares. São Paulo: Atheneu; 2002. p.179-86.

4. Almeida JM, Luz SAB, Ued FV. Support of breast feeding by health professionals: integrative review of the literature. Rev Paul Pediatr. 2015;33(3):355-62. DOI: https://doi.org/10.1016/j.rpped.2014.10.002

5. World Health Organization. Report of an expert consultation on the optimal duration of exclusive breastfeeding. Geneva: World Health Organization; 2001.

6. Machado MCM, Assis KF, Oliveira FDCC, Ribeiro $A Q$, Araújo RMA, Cury AF et al. Determinants of the exclusive breast feeding abandonment: psychosocial factors. Rev Saude Pub. 2014;48(6):985-94. DOI: https://doi.org/10.1590/S0034$\underline{8910.2014048005340}$

7. Silva NM, Waterkemper R, Silva EF, Cordova FP, Bonilha ALL. Conhecimento de puérperas sobre amamentação exclusiva. Rev Bras Enferm. 2014;67(2):290-5.

DOI: http://dx.doi.org/10.5935/0034-7167.20140039

8. Bueno MB, Souza JMP, Souza SB, Paz SMRS, Siqueira AAF. Riscos associados ao processo de desmame entre crianças nascidas em hospital universitário de São Paulo entre 1998 e 1999: estudo de coorte prospectivo do primeiro ano de vida. Cad Saúde Pública. 2003;19:1453-60. DOI: https://doi.org/10.1590/S0102311X2003000500024

9. Pérez-Scamilla R, Lutter C, Segall AM, Rivera A, Trevino-Siller $S$, Sanghvi $T$. Exclusive breastfeeding duration is associated with attitudinal, socioeconomic and biocultural determinants in three latinamerican countries. J Nutr.
$1995 ; 125: 2972-84$.

DOI:

http://dx.doi.org/10.1590/S0104-

$\underline{42302007000600020}$

10. Santos KK, França-Botelho AC. Mitos que podem prejudicar o aleitamento materno em Perdizes, MG. Saúde Pesq. 2010;3(2):139-47.

11. Silva ACC, Conceição CS, Watanabe RTM. Incentivo e promoção do aleitamento materno em grupo de gestantes. Anais Semex. 2016;8.

Recebido para publicação em 14/08/2017

Revisado em 08/03/2018

Aceito em 03/07/2018 